\title{
INTEGRAÇÃO ENSINO, PESQUISA E EXTENSÃO AGROFLORESTAL NA AMAZÔNIA CENTRAL
}

Breno Pinto Rayol Fabrízia de Oliveira Alvino-Rayol

Resumo: O objetivo do presente trabalho é discorrer sobre a importância das ações extensionistas integradas com atividades de ensino e pesquisa no contexto agroflorestal, visando à construção participativa de alternativas promissoras a agricultura familiar na Amazônia. A partir das demandas identificadas pelas atividades de pesquisa-ação foram articuladas atividades acadêmicas de ensino para o desenvolvimento de ações extensionistas usando metodologia participativa. As observações relatadas reforçam o papel da indissociabilidade entre ensino, pesquisa e extensão e a sua importância para a transição agroecológica. O trabalho destacou que a integração dos diferentes atores sociais é indispensável para a construção do conhecimento agroflorestal. A articulação de atividades acadêmicas com as demandas da comunidade gera benefícios à universidade e à sociedade em geral.

Palavras chave: Educação; Extensão; Agrofloresta; Agricultura Familiar.

Abstract: The objective of the present study is to discuss the importance of integrated extension activities with teaching and research activities in the agroecological context, aiming at the participatory construction of promising alternatives to family agriculture in the Amazon. From the demands identified by action-research activities, academic teaching activities were articulated for the development of extensionist actions using participatory methodology. The observations reported reinforce the role of the inseparability between teaching, research and extension and their importance for the agroecological transition. The work highlighted that the integration of different social actors is indispensable for the construction of agroecological knowledge. The articulation of academic with the demands of the community generates benefits to the university and society in general.

Keywords: Education; Extension; Agroforest; Family farming.

\section{INTRODUÇÃO}

Os modelos de desenvolvimento agrícolas vinculados essencialmente às finalidades econômicas vêm provocando fortes impactos sociais e ambientais, tais como: perda acelerada de biodiversidade, uso inadequado e contaminação de recursos naturais (principalmente água, solo e ar), desmatamentos, queimadas, êxodo rural e destruição de arranjos produtivos locais e insegurança alimentar (NAIR, 2007; NAIR, 2008; MACHADO et al., 2008). Neste contexto, uma das alternativas para melhorar esta realidade é adoção de sistemas de produção que sejam adequados para as diferentes condições ambientais. Inspirada na cobertura permanente que caracteriza a estrutura arbórea da floresta tropical, uma alternativa sustentável promissora de produção primária é a implantação dos diferentes modelos de sistemas agroflorestais (SAF’s). Esses sistemas diversificados de produção são caracterizados pelo cultivo simultâneo ou 
escalonado, no espaço e no tempo, de árvores com espécies agrícolas anuais, plurianuais ou perenes, na presença ou não de animais (DUBOIS, 1996; NAIR, 2007). Portanto, os sistemas agroflorestais, quando baseados em princípios agroecológicos, podem ser utilizados como estratégias para contribuir com o desenvolvimento sustentável (PALUDO e COSTABEBER, 2012).

A agroecologia possui uma abordagem holística e complexa, além disso, procura valorizar os conhecimentos e saberes tradicionais integrando-os às tecnologias heterogêneas adequadas às realidades locais e à cultura de comunidades rurais (CAPORAL et al., 2006). Porém apesar das vantagens da agroecologia, ainda existem muitos desafios para a extensão rural agroecológica, que vão além de simples mudanças de modelos tecnológicos, incluem, portanto, mudanças de velhos paradigmas (SILIPRANDI, 2002). Portanto, formas diferenciadas do processo de ensino-aprendizagem são essenciais no enfoque agroecológico, devendo ser superadas as práticas pedagógicas convencionais, bem como matrizes disciplinares fragmentadas (CAPORAL et al., 2006).

Neste contexto, o objetivo do presente trabalho é mostrar a importância das ações extensionistas integradas com atividades de ensino e pesquisa no contexto agroflorestal, visando a construção participativa de alternativas agroecológicas promissoras a agricultura familiar da Amazônia.

\section{METODOLOGIA}

Com a finalidade de contribuir com desenvolvimento de estratégias adaptadas à realidade dos agricultores familiares para a viabilização do potencial sócio-econômico e ecológico de sistemas produtivos sustentáveis, o projeto "O uso de sistemas agroflorestais como alternativa promissora à agricultura familiar do Baixo Amazonas, oeste do Pará" foi conduzido pela Universidade Federal do Oeste do Pará (UFOPA) em cooperação com a Empresa de Assistência Técnica e Extensão Rural do Pará (EMATER-PA) e Instituto de Educação, Ciência e Tecnologia do Pará (IFPA) com o apoio financeiro da Fundação de Amparo a Pesquisa Amazônia Paraense (FAPESPA). O projeto envolveu uma série de ações integradas entre ensino, pesquisa e extensão, em caráter multidisciplinar e participativo levando em consideração as experiências e o conhecimento empírico dos agricultores e agricultoras familiares envolvidos.

O presente trabalho foi realizado em três municípios do Baixo Amazonas: Belterra, Mojuí dos Campos e Santarém. As ações de pesquisas foram realizadas em propriedades de agricultores familiares que integram o conceito de sistemas agroflorestais em seu meio de 
produção. As propriedades foram identificadas por meio de informações dos técnicos da Empresa de Assistência Técnica e Extensão Rural do Pará (EMATER-PA) e organizações não governamentais atuantes na região (ONGs).

A partir das demandas identificadas pelas atividades de pesquisa-ação (THIOLLENT, 2005), articulou-se o desenvolvimento das atividades acadêmicas (ensino) integradas às de extensão. As ações de ensino foram implementadas nas disciplinas: a) Agrossilvicultura no curso de graduação em engenharia florestal (UFOPA) (Figura 1); b) Sistemas agroflorestais na especialização em Agroecologia (UFOPA); c) Produção de Mudas e sementes e Sistemas agroflorestais no curso técnico em agropecuária (IFPA). O trabalho envolveu ações de diagnóstico, análise e proposição de alternativas agroflorestais que foram discutidas e construídas pelos estudantes e agricultores e agricultoras familiares envolvidos (Figura 2). Os saberes, conhecimentos e experiências dos próprios produtores nortearam os métodos e técnicas agroflorestais permitindo o fortalecimento das suas capacidades de experimentação e inovação.

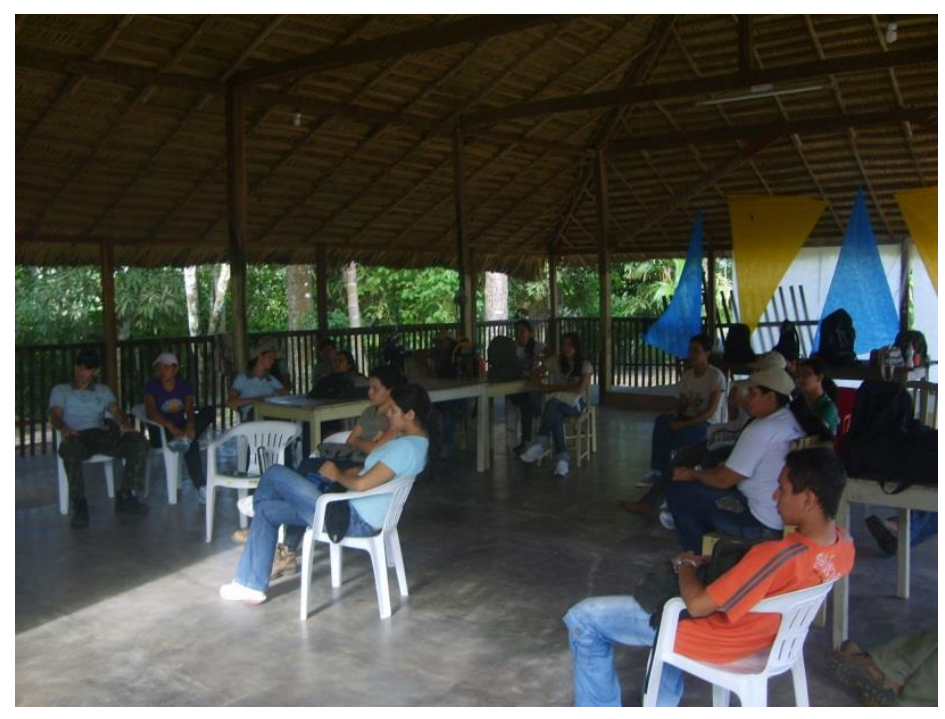

Figura 1 - Aula prática de Agrossilvicultura com discentes de Engenharia Florestal em área de agricultura família, Santarém, Pará. 


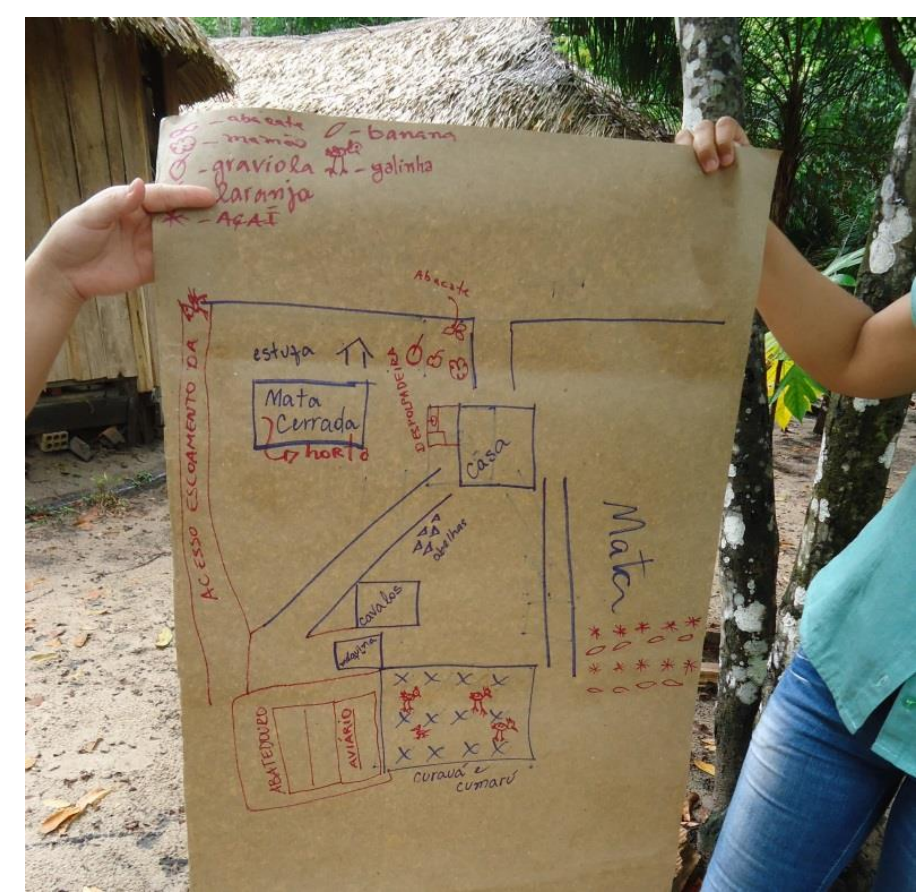

Figura 2 - Apresentação de alternativas agroecológicas discutidas e construídas pelos estudantes e agricultores e agricultoras familiares envolvidos durante as ações, Santarém, Pará.

Fonte: Acervo fotográfico dos pesquisadores (2018)

\section{RESULTADOS E DISCUSSÃO}

Um total de 259 pessoas participou desse processo de construção de conhecimento. Destas, um pouco menos da metade eram mulheres (42\%), observando certo equilíbrio na questão de gênero (Figura 3). A maioria dos participantes foram produtores familiares (Figura 4). A heterogeneidade de perfil de participantes das ações é um aspecto positivo a ser relatado, pois a pluralidade de conhecimentos e saberes proporcionou uma maior riqueza de experiências vivenciadas. Isso é um exemplo de como a interdisciplinaridade contribui para transcender a convencional dicotomia teoria/prática (PUHL e DRESCH, 2016). 


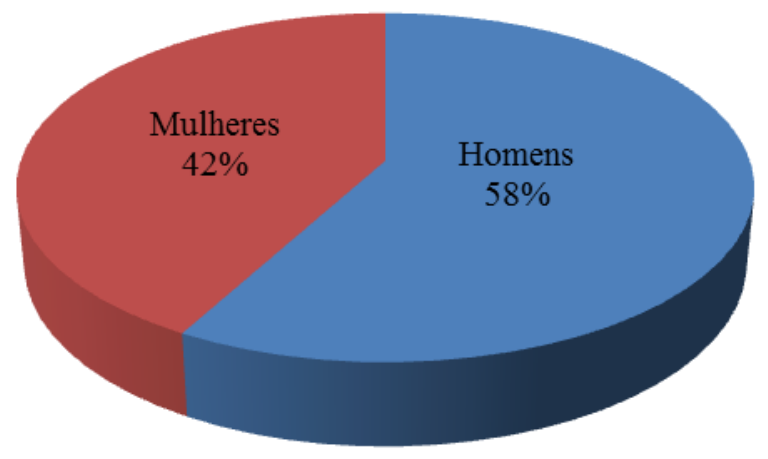

Figura 3 - Gênero dos participantes das atividades de extensão desenvolvidas Fonte: Dados dos pesquisadores (2018)

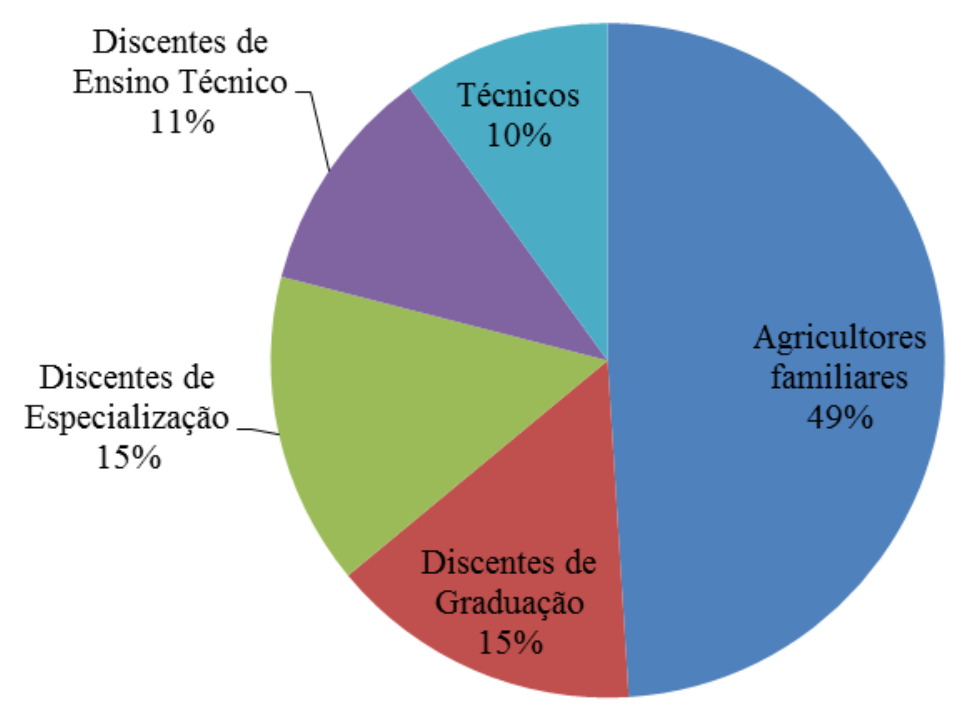

Figura 4 - Perfil dos participantes das atividades desenvolvidas Fonte: Dados dos pesquisadores (2018) 
O campo ao ser utilizado como espaço didático, por meio da socialização de saberes oriundos de diferentes fontes possibilitou, especialmente aos discentes, a valorização dos conhecimentos tradicionais dos agricultores e agricultoras familiares, deixando-os mais próximos da realidade local. A articulação entre os diversos saberes é fundamental para se pensar na indissociabilidade pesquisa-extensão-ensino (MOITA e ANDRADE, 2009). As atividades de extensão integradas e articuladas com as de ensino e pesquisa proporcionaram uma potencialização na construção do conhecimento. As experiências vividas pelos discentes favoreceram a formação de uma visão crítica e um maior entendimento da realidade da agricultura familiar da região. É papel da universidade oportunizar aprendizagem de um tipo de conhecimento socialmente contextualizado a fim de contribuir com a formação de um sujeito autônomo e comprometido com a coletividade (PIVETTA et al., 2010).

O processo de ensino-aprendizagem relatado foi além dos muros da universidade, trouxe, portanto, benefícios múltiplos, não só para os discentes e produtores familiares envolvidos, mas para a sociedade como um todo que receberá profissionais críticos e reflexivos conscientes de suas responsabilidades sociais. A formação acadêmica quando focada somente no ensino, ou seja, dissociada da pesquisa extensão, prepara o profissional somente de forma técnica para o mercado de trabalho, e muito pouco contribui com a sua formação social (MAZZILLI, 2011), portanto, a integração do ensino, da pesquisa e extensão exige repensar paradigmas e modelos de pensamentos de visão tradicional, com vistas a diminuir a distância entre a universidade e a comunidade (PUHL e DRESCH, 2016).

Esse processo vai ao encontro da formação de extensionistas com um novo perfil, citada por Caporal e Costabeber (2000), onde esses estarão preparados para compreender a importância dos saberes e conhecimentos dos produtores e produtoras familiares para o desenvolvimento de uma prática social visando a gestão dos agroecossistemas numa perspectiva de desenvolvimento sustentável das comunidades.

Atualmente, ainda é muito comum projetos proporem ações extensionistas sem ter conhecimento da real demanda das comunidades com as quais se propõem trabalhar. A visão meramente difusionista e tecnicista contribui muito pouco com o desenvolvimento da agricultura familiar.

A análise das experiências aqui relatadas permitiu constatar que a pesquisa-ação foi indispensável para a construção do conhecimento agroecológico e agroflorestal na região amazônica, proporcionando uma base sólida na execução das ações através da participação e a integração de diferentes atores sociais (agricultores/ agricultoras familiares, discentes, técnicos e docentes). 
Os agricultores e agricultoras com seus saberes e conhecimentos foram de fundamental importância na construção desse processo. Atuaram como experimentadores e contribuíram muito com adaptações de técnicas as suas realidades. Ressalta-se, portanto, a importância em fortalecer ações para que o ensino não se torne tão distante da realidade local (MOITA e ANDRADE, 2009).

A articulação de atividades acadêmicas focadas em suprir as demandas da comunidade mostrou ser uma estratégia exitosa por contribuir com:

- Estímulo e fortalecimento da experimentação e inovação por parte de agricultores e agricultoras familiares;

- Formação de um novo perfil de profissionais comprometidos com a realidade local, devido às vivências na agricultura familiar;

- Enfraquecimento da visão fragmentada e limitada das disciplinas na academia;

No projeto também foram realizadas outras ações extensionistas, tais como, dias-decampo, visitas técnicas, palestras, seminários e minicursos (Figuras 5 e 6). Ressalta-se que para todas essas ações foi utilizada como método de construção coletiva do conhecimento a troca de experiências por meio de uma abordagem sistêmica.

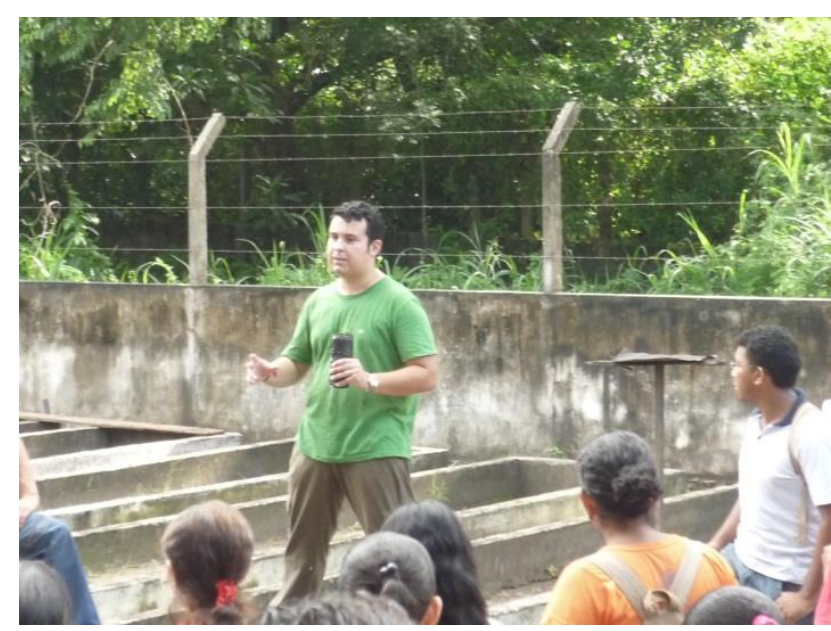

Figura 5- Minicurso de Produção de Mudas para Sistemas Agroflorestais, Santarém, Pará. 


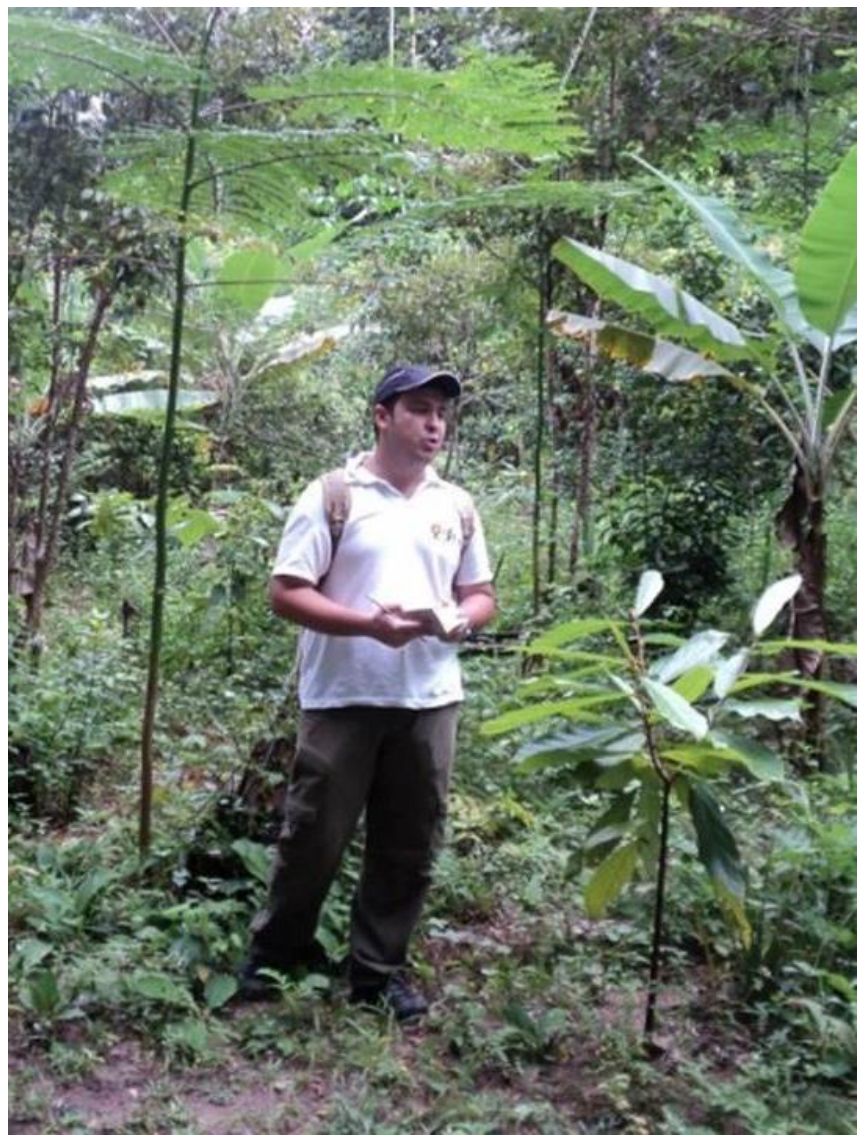

Figura 6- Dia de campo sobre sistemas agroflorestais em área de agricultura familiar, Santarém, Pará.

Fonte: Acervo fotográfico dos pesquisadores (2018)

\section{CONSIDERAÇÕES FINAIS}

As observações relatadas reforçam o papel da indissociabilidade entre ensino, pesquisa e extensão. O trabalho destacou que a integração dos diferentes atores sociais é indispensável para a construção do conhecimento agroflorestal. A articulação de atividades acadêmicas com as demandas da comunidade gera benefícios à universidade e à sociedade em geral.

Essa prática formativa relatada, por estreitar os vínculos da academia com a comunidade, contribuiu com a aproximação da academia com a realidade social amazônica. A estratégia ao estimular a interação de estudantes com agricultores e agricultoras familiares proporcionou oportunidade de uma formação crítico-reflexiva pautada na valorização da diversidade de saberes e que pode vir a contribuir com o fortalecimento da formação integral de profissionais capacitados tecnicamente, mas também com valores éticos e comprometidos com transformações sociais. 


\section{AGRADECIMENTOS E APOIOS}

À Fundação Amazônia Paraense de Amparo à Pesquisa (FAPESPA) pelo apoio financeiro; Às agricultoras e agricultores familiares pela cordialidade com que nos receberam em suas propriedades; Aos discentes da Universidade Federal do Oeste do Pará (UFOPA) e do Instituto de Educação, Ciência e Tecnologia do Pará (IFPA) e técnicas/técnicos da Empresa de Assistência Técnica e Extensão Rural do Pará (EMATER-PA) pela participação nas atividades.

\section{REFERÊNCIAS BIBLIOGRÁFICAS}

CAPORAL, F. R.; COSTABEBER, J. A. Agroecologia e desenvolvimento rural sustentável: perspectivas para uma nova extensão rural. Agroecologia e Desenvolvimento Rural Sustentável, v.1, n.1, p.16-37, 2000.

CAPORAL, F. R.; COSTABEBER, J. A.; PAULUS, G. Agroecologia: matriz disciplinar ou novo paradigma para o desenvolvimento rural sustentável. In: CONGRESSO BRASILEIRO DE AGROECOLOGIA, 3., 2006, Florianópolis. Anais... Florianópolis : ABA, 2006. 25p.

DUBOIS, J.C.L. (Org.). Manual agroflorestal para a Amazônia. Volume 1. Rio de Janeiro: REBRAF, 1996.

MACHADO, A.T.; SANTILLI, J.; MAGALHÃES, R. A agrobiodiversidade com enfoque agroecológico: implicações conceituais e jurídicas. Brasília, DF: Embrapa Informação Tecnológica, 2008. 98p. (Texto para Discussão, 34).

MAZZILLI, S. Ensino, pesquisa e extensão: reconfiguração da universidade brasileira em tempos de redemocratização do Estado. RBPAE, v.27, n.2, p. 205-221, 2011.

MOITA, F.M.G.S.C; ANDRADE, F.C.B. Ensino-pesquisa-extensão: um exercício de indissociabilidade na pós-graduação. Revista brasileira de educação, v. 14, n. 41, p. 269-280, 2009.

NAIR, P. K. The coming of age of agroforestry. Journal of the Science of Food and Agriculture, v. 87, n. 9, p. 1613-1619, 2007.

NAIR, P.K. Ramachandran et al. Agroecosystem management in the 21st century: it is time for a paradigm shift. Journal of Tropical Agriculture, v. 46, n. 1-2, p. 1-12, 2008.

PALUDO, R.; COSTABEBER, J. A. Sistemas agroflorestais como estratégia de desenvolvimento rural em diferentes biomas brasileiros. Revista Brasileira de Agroecologia, v.7, n.2, p.63-76, 2012.

PIVETTA, H.M.F.; STEIN BACKES, D.; CARPES, A.; HOLANDA TAVARES

BATTISTEL, A. L.; MARCHIORI, M. Ensino, pesquisa e extensão universitária: em busca de uma integração efetiva. Linhas Críticas, v.16, n.31, p.377-390. 2010. 
PUHL, M. J.; DRESCH, O. I. O princípio da indissociabilidade entre ensino, pesquisa e extensão e o conhecimento. Di@logus, v.5, n.1, p.37-55, 2016.

SILIPRANDI, E. Desafios para a extensão rural: o" social" na transição agroecológica. Agroecol. Desenvolv. Rur. Sustent, v.3, n.3, p.38-48, 2002.

THIOLLENT, M. Metodologia da pesquisa-ação. 14.ed. aumentada. São Paulo: Cortez, 2005. 